\title{
Short limbed dwarfism, genital hypoplasia, sparse hair, and vertebral anomalies: a variant of Ellis-van Creveld syndrome?
}

\author{
Jean-Pierre Fryns, Philippe Moerman
}

\begin{abstract}
A male newborn with acromesomelic short limbed dwarfism, genital hypoplasia, and vertebral anomalies is reported. As the child had an important number of clinical and radiological symptoms seen in patients with Ellis-van Creveld syndrome, we raise the question of whether he may represent a variant example of this syndrome despite the absence of cardinal symptoms such as postaxial polydactyly and ectodermal changes (nail hypoplasia).

(f Med Genet 1993;30:322-4)
\end{abstract}

\section{Case report}

The proband, a boy, was the first child of healthy, unrelated parents with no relevant family history. The father was 27 and the mother 24 years old. Pregnancy and delivery, at 39 weeks, were normal. Birth weight was $2180 \mathrm{~g}$, length $42 \mathrm{~cm}$, and head circumference $33 \mathrm{~cm}$. Immediately after birth major cardiorespiratory difficulties were noted and a number of dysmorphic signs became progressively evident. The head (fig 1) was relatively large and square with a high and broad forehead and large anterior fontanelle. There was marked ptosis of the left eyelid and fixed contraction of the left pupil. A naevus flammeus extended over the base of the nose, the mid-forehead, and both eyelids. The nose was small with upturned nares and the philtrum short with thin lips. Scalp hair was sparse and thin. The chin was relatively small and the neck short with slight posterior webbing. The frenulum of the upper lip was prominent and adherent to the alveolar ridge. There was almost complete fusion between the upper lip and gum and the alveolar ridges were broad and irregular. There was marked bodily disproportion with shortening of the upper and lower limbs, somewhat more evident in the lower legs. The hands and feet were short and broad with bilateral simian creases and proximal implantation of both fifth toes. The upper thorax was small and there was a rudimentary extra nipple $2 \mathrm{~cm}$ below the left nipple. The genitalia were small with a short $(2 \mathrm{~cm})$ phallus and hypoplastic scrotum without palpable testes. The child failed to thrive and died at the age of 4 months (weight $3145 \mathrm{~g}$, length $50.5 \mathrm{~cm}$, head circumference $37.2 \mathrm{~cm}$, foot length $74 \mathrm{~mm}$ ) after chronic cardiac decompensation.

Routine biochemical, metabolic, and immu-

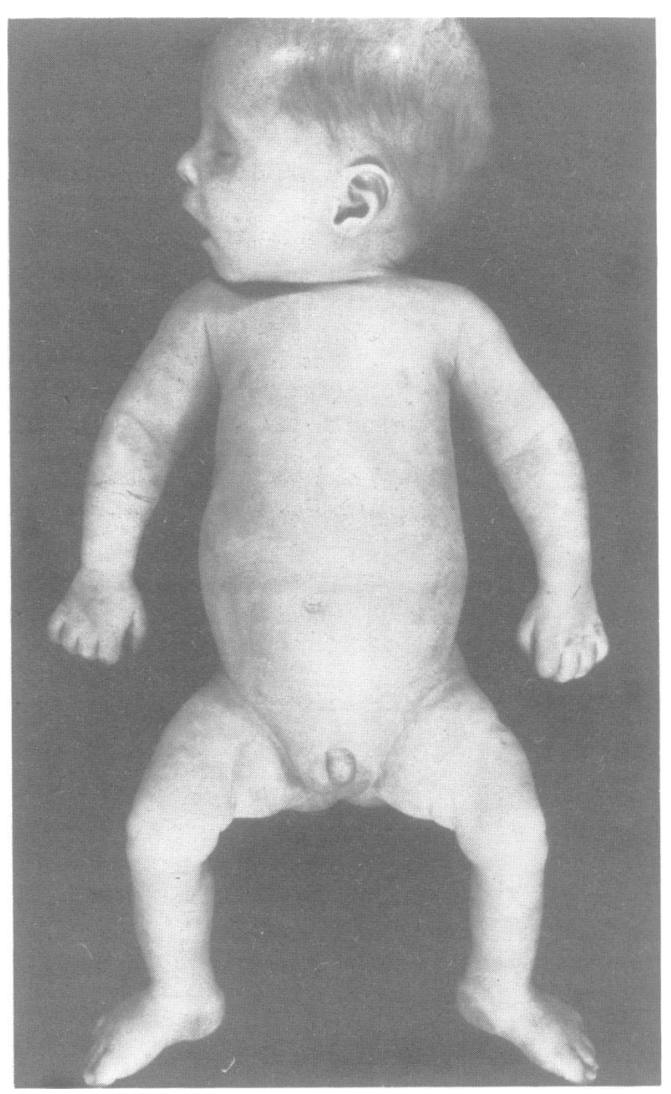

Figure 1 General appearance of the proband.

nological examinations were normal. Chromosomal analysis showed a $46, \mathrm{XY}$ normal male karyotype in a peripheral blood lymphocyte culture and a fibroblast culture from a postmortem skin biopsy.

Pre- and postmortem skeletal $x$ rays showed a narrow thorax with short horizontal ribs and multiple cervical and thoracic fusion defects and segmentation anomalies (fig 2). The 12th pair of ribs was very small with only rudimentary development on the left. The iliac wings were flared and hypoplastic; there was a trident deformity of the acetabular roofs and the sciatic notches were small. The tubular bones were short, heavy, and mildly bowed. There was lack of ossification of the epiphyses in the knee region and two ossification centres in each tarsal region. The metaphyses were broad and somewhat irregular with bony spikes at the distal metaphyses of the femora (fig 3), the medial side of the proximal metaphysis of the tibiae, and the distal metaphyses of the humeri. The metacarpals and metatarsals were short with relatively longer second metacarpals. The middle phalanges were especially short and the distal phalanges rudimentary (fig 


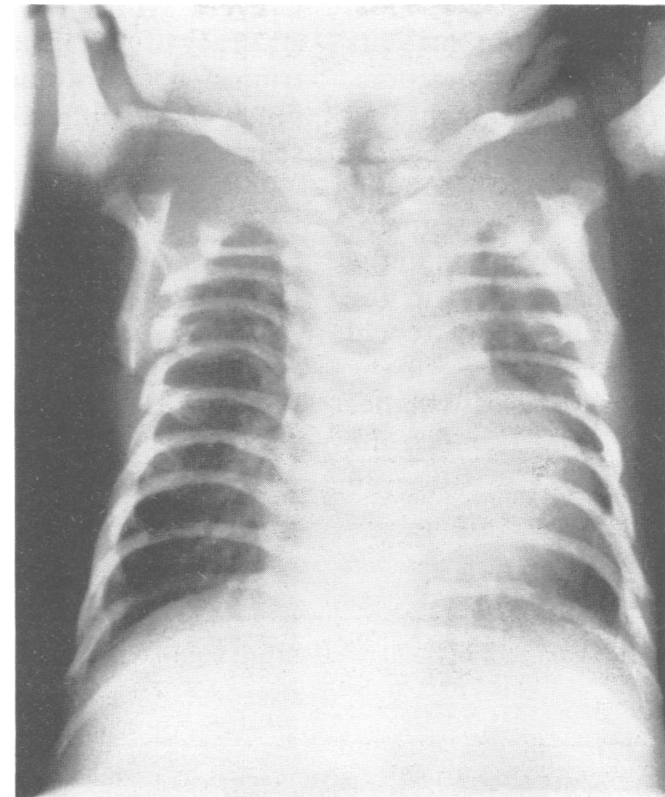

Figure $2 \boldsymbol{X}$ rays of the narrow thoracic cage with shor horizontal ribs and multiple cervical and thoracic fusion defects and segmentation anomalies.

4). Histological examination of the enchondral ossification showed no distinct anomalies. Necropsy showed a complex cardiopathy with a double superior vena cava, absent sinus coronarius, left cor triatrum, and incomplete persistent atrioventricular canal.

Macroscopic examination of the brain showed a paramedian cystic structure $(3.5 \mathrm{~cm}$ in diameter) in the left occipital region extending into the cisterna venae cerebri magnae.

\section{Discussion}

This malformed male infant presented an unusual combination of clinical signs and

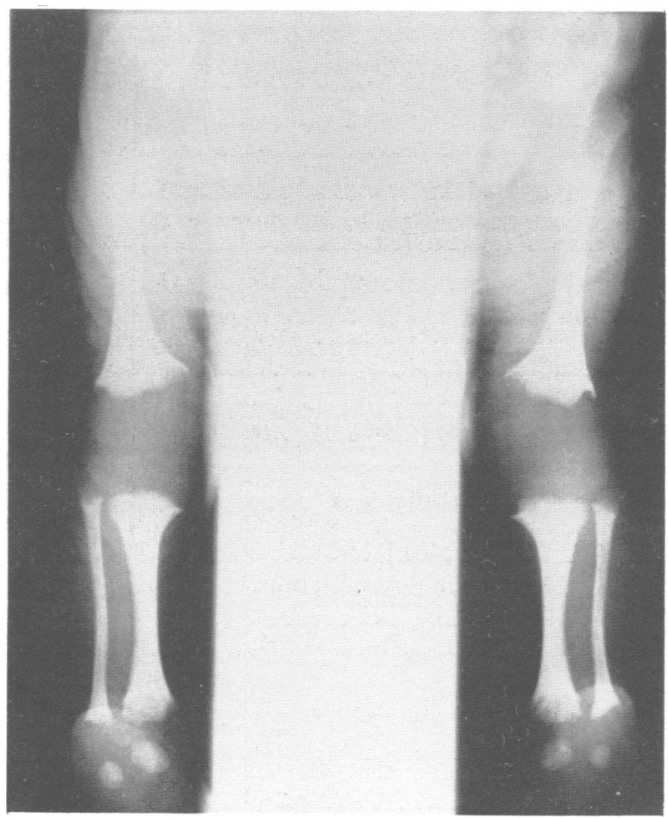

Figure 3 Broad and somewhat irregular metaphyses with bony spikes at the distal metaphyses of the femora and the medial side of the proximal metaphyses of the tibia. There is lack of ossification of the epiphyses in the knee region and two ossification centres are present in the tarsal region. symptoms and caused us a considerable problem in the final diagnosis and genetic counselling of these young parents. An extensive search of published reports on the association of short limbed acromesomelic dwarfism, vertebral fusion and segmentation anomalies, genital hypoplasia, AV canal, and CNS malformations gave no conclusive suggestions for a final diagnosis.

Sussman et al $^{1}$ reported two sibs (one male, one female) with short limbed dwarfism, lack of segmentation of the thoracolumbar vertebrae, and metaphyseal modelling abnormalities. An ASD and cardiomyopathy were present in the first sib who died at 6 months. The facial stigmata, however, were different and CNS abnormalities were not present.

Looking in detail at the spectrum of clinical and radiological anomalies present in this child we found a considerable overlap with an important number of symptoms of Ellis-van Creveld syndrome ${ }^{23}$ including short limbed acromesomelic dwarfism, midfacial naevus flammeus, sparse hair, congenital heart disease with double superior vena cava, left cor triatrum and incomplete persistent atrioventricular canal, fusion between the upper lip and gum, genital hypoplasia, CNS malformation with cystic structure in the left occipital region extending into the fossa posterior, shortness of ribs with hypoplasia of the 12th pair, lack of ossification of the epiphyses in the knee region, tarsal ossification, and bony spikes at the

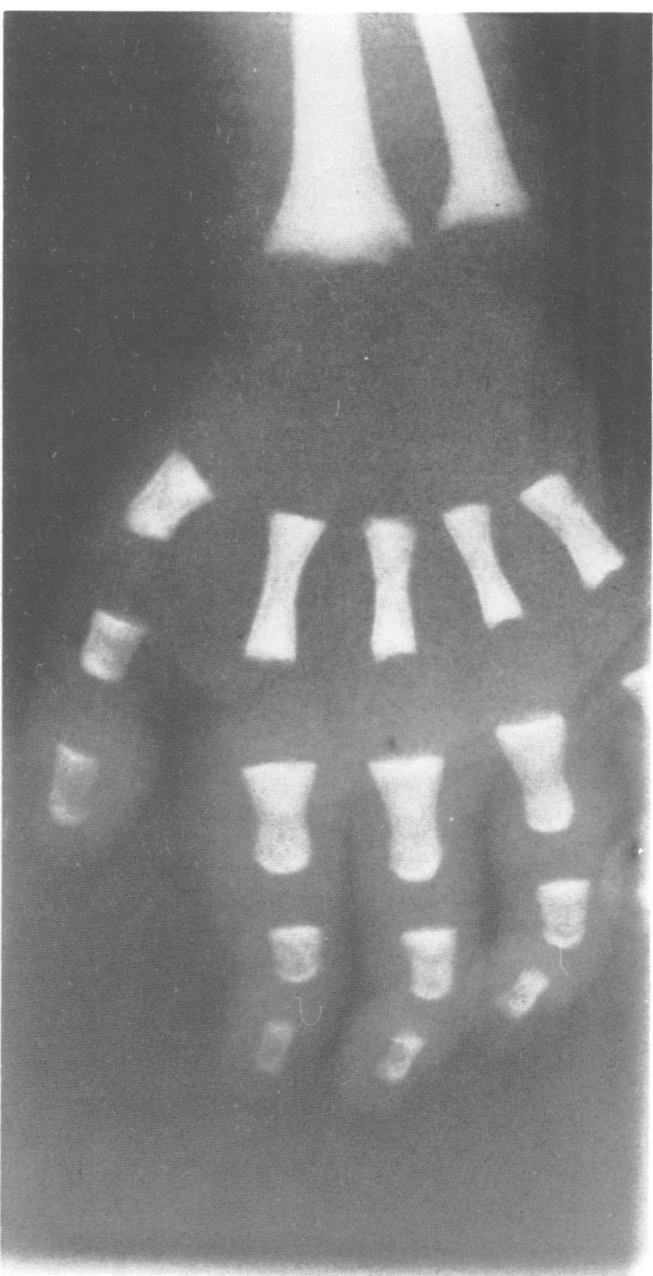

Figure $4 X$ ray of the left hand showing acromesomelic shortening. 
femoral, tibial, and humeral metaphyseal ends. A number of symptoms present in patients with classical Ellis-van Creveld syndrome, however, were absent in our patient, including bilateral postaxial polydactyly of the hands, and ectodermal dysplasia affecting the nails and teeth. Moreover, vertebral fusion segmentation anomalies, an important symptom in the present child, have not been reported in the spectrum of anomalies of Ellis-van Creveld syndrome.

We therefore raise the question of whether the findings in the present child broaden the spectrum of phenotypic findings in Ellis-van
Creveld syndrome. If so, the feasibility and accuracy of prenatal diagnosis based on second trimester ultrasound and fetoscopy may be much more difficult than considered at present.

1 Sussman MD, Kelly T, Rosenbaum KN, Balian G. Abnormality of cartilage collagen in a patient with unclassified chondrodystrophy. $\mathcal{f}$ Orthop Res 1984;2:339-45.

2 Gorlin RJ, When MM, Nevin SJ. Ellis-Van Creveld syndrome (chondroectodermal dysplasia). In: Syndromes of the neck and head. 3rd ed. Oxford Monographs on Medical Genetics No 19. New York, Oxford: Oxford University Press, 1990.

3 Taybi H, Lackman RS. Chondroectodermal dysplasia. In: Radiology of syndromes, metabolic disorders and skeletal dysplasias. 3rd ed. Chicago: Year Book Medical Publishers, 1990. 